\title{
Solitons in Nuclear Time-Dependent Density Functional Theory
}

\author{
Yoritaka Iwata* \\ Faculty of Chemistry, Materials and Bioengineering, Kansai University, Osaka, Japan
}

The soliton existence in sub-atomic many-nucleon systems will be discussed. In many nucleon dynamics represented by the nuclear time-dependent density functional formalism, much attention is paid to energy and mass dependence of the soliton existence. In conclusion, the existence of nuclear soliton is clarified if the temperature of nuclear system ranges from 10 to $30 \mathrm{MeV}$. With respect to the mass dependence ${ }^{4} \mathrm{He}$ and ${ }^{16} \mathrm{O}$ are suggested to be the candidates for the self-bound states exhibiting the property of nuclear soliton.

Keywords: TDDFT, multi-dimensional solition, full skyrme interaction, many-nucleon system, ion collisions

\section{INTRODUCTION}

The concept of nuclear soliton is proposed by its existence in the three-dimensional nuclear time-dependent density functional formalism. The solitons in this article are the waves stably traveling without changing shape and velocity even after collisions between waves (Figure 1). In this sense, as for the terminologies of classical and quantum field theory, what we study in this article is not similar to the topological soliton [1,2], but rather corresponds to the non-topological soliton [3]. From here on, we refer simply to "soliton" for a kind of non-topological soliton. The mathematically common property of a soliton (for example, see Ablowitz [4]) has been clarified as

Reviewed by:

Giuseppe Verde, National Institute for Nuclear Physics,

*Correspondence: Yoritaka Iwata iwata_phys@08.alumni.u-tokyo.ac.jp

Specialty section:

This article was submitted to Nuclear Physics, a section of the journal Frontiers in Physics

Received: 04 February 2020 Accepted: 15 April 2020

Published: 30 June 2020

Citation:

Iwata Y (2020) Solitons in Nuclear Time-Dependent Density Functional

- Non-linearity

- Dispersive property

being independent of the size and medium of wave. The common properties of solitons are essential to the solitons existence, and several uncommon properties specific to nuclear solitons such as

- Quantum effect with the fermi statistics

- Many-body effect leading to the collectivity

can modify the conditions of soliton existence, where a competition between them possibly appears. In most soliton research mentioned here, the size and model dependent additional properties are not seriously considered. Here we employ the nuclear time-dependent density functional theory (TDDFT) in which all the above four properties are included in a self-consistent manner. In particular the collectivity of many-nucleon systems has been successfully treated by the nuclear DFT with and without time-dependence (for example, see Greiner and Maruhn [5]).

The solitons are observed in any scale, if the mathematically common property is held by the master equation. This fact has something to do with the size and model dependence of the two common properties. The nuclear soliton is found in sub-atomic femto-meter scales whose energy is at the order of $\mathrm{MeV}$ (mega electron volt). Such a specific scale arises from the effective unit of motion: the nucleon degree of freedom in the case of a nuclear soliton. For example, the effective unit of motion for the optical soliton is the photon. In other words, as is known in nuclear physics, the motion of the nucleus at the energy order of $\mathrm{MeV}$ is governed by the independent nucleon motion (for example, see Ring and Schuck [6]). 


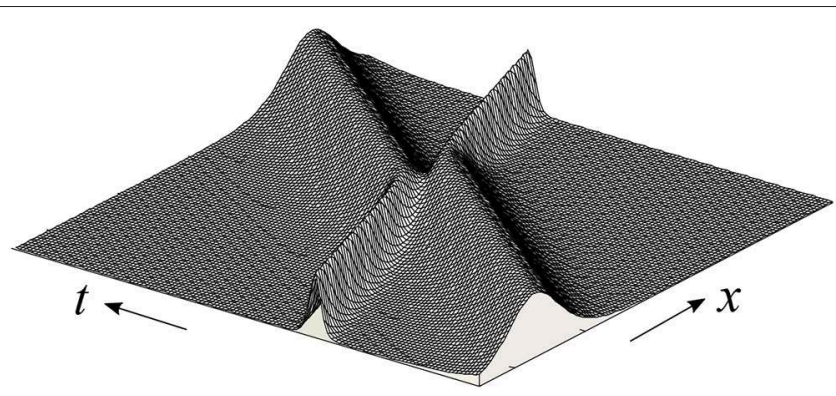

FIGURE 1 | Two soliton solution of KdV equation $(\alpha=1)$. Although the momentum and shape are exactly conserved, time delay appears due to the collision (around $(t, x)=(0,0))$.

The soliton is a wave with both individuality and stability. On the other hand, the nuclear soliton is also regarded as bringing about a nuclear matter state with perfect fluidity. It is worth mentioning here that perfect fluidity can be rephrased as inertness in the context of reaction theory. Accordingly, the nuclear soliton is expected to be associated with some important physics if its existence is established. Indeed, perfect fluidity leads to the conservation of the number of vortexes. Since celestial bodies consist of nuclear matter, the quantitative understandings of the nuclear soliton are able to show a new aspect of the matter/heat transportation inside the (compact) stars. Furthermore, perfect fluidity is associated with the dissipation property of low-energy heavy-ion collisions that has been a long standing open problem in microscopic nuclear reaction theory. Perfect fluidity is also associated with the conservation of nuclear matter without the loss of any information: i.e., isentropic property arising from the time-reversal symmetry [7]. As the conservation property of the soliton has already been utilized in the optical fiber, the preservation property of nuclear matter is expected to be utilized in the nuclear engineering for preserving and condensing a certain projectile nucleus. In particular, the well-preserved nuclear matter is expected to be used for the reduction of nuclear waste by the nuclear transmutation, with the extremely high intensity/density projectile of reactions, which is not only to make a high intensity/density beam but also high projectile-density matter in the nuclear reactor.

This article is organized as follows. The basic concepts of wave propagation are introduced in section 2 . The general definition of solitary wave and soliton is shown in section 3 . The existence of nuclear soliton is discussed in section 4 . The summary and perspectives are presented in section 5 .

\section{EQUATION OF WAVES}

This section is devoted to introducing the basic concepts for wave propagation, which provides a working area of the soliton research. For the purpose of introducing the concept of dispersive property, we begin with the linear wave:

$$
u(t, x)=A \exp (i(k x-\omega t+\alpha))
$$

in one-dimensional space, where $k$ means the wave number, $\omega$ the angular frequency, and $\alpha$ the phase. This wave is also referred to as the plane wave in the multi-dimensional case, and to a traveling wave in more general fields. The first order linear hyperbolic equation (advection equation) is written by

$$
\partial_{t} u+c \partial_{x} u=0
$$

in one-dimensional space $\mathbb{R}$, where $c$ is a real constant, meaning the propagation speed. It is well-known that this equation holds the solution represented by the d'Alembert's formula, so that the plane wave (1) satisfies this equation. The linear dispersion relation $\omega=c k$ is satisfied by the plane wave solution. The plane wave solution can also be associated with the second order linear wave equations, more closely to the present interest, the Klein-Gordon equation:

$$
\partial_{t}^{2} u-c^{2} \partial_{x}^{2} u+\left(\frac{m c^{2}}{\hbar}\right)^{2} u=0
$$

describing a quantum scalar or pseudoscalar fields [8]. By considering the same plane wave solution, another relation $\omega^{2}=$ $c^{2}\left(k^{2}+m^{2} c^{2} / \hbar^{2}\right)$ is obtained, which is asymptotically equal to $\omega= \pm c k$ (Figure 2). Note that the dispersion relation in the massless case $(m=0)$ also becomes $\omega= \pm c k$.

The Schrödinger equation is known to describe the nonrelativistic quantum physics. The linear dispersion relation $\omega=$ $c k$ is violated in case of Schrödinger type waves. On the other hand, it is readily confirmed that the plane-wave solution is also the solution of the linear Schrödinger equation:

$$
i \partial_{t} u+c \partial_{x}^{2} u=0
$$

in one-dimensional space $\mathbb{R}$, where $c$ is a real constant being represented by $c=-\hbar / 2 m$ using the Dirac constant $\hbar$ and the mass $m$. In this case another dispersion relation $\omega=c k^{2}$ is satisfied instead. Such waves, without satisfying the linear dispersion relation $\omega=c k$, are called the dispersive wave. It is worth noting here that the non-relativistic approximation of Klein-Gordon equation corresponds to the Schrödinger equation. As a result, the Schrödinger equation is a typical example of dispersive wave equations.

\section{NON-LINEAR DISPERSIVE WAVES}

\subsection{Korteweg-de Vries Equation}

The concepts of solitary wave and soliton are introduced. For verifying the soliton existence in sub-atomic quantum equations, we focus on two relevant equations: the Korteweg-de Vries equation and non-linear Schrödinger equation. These equations are not only dispersive wave equations but also non-linear evolution equations.

First, in the flow of shallow water, the concept of a solitary wave was introduced by Scott-Russel [9] in 1844. Indeed, they observe that

- A single wave moves stably on the flat surface without changing the shape and velocity. 


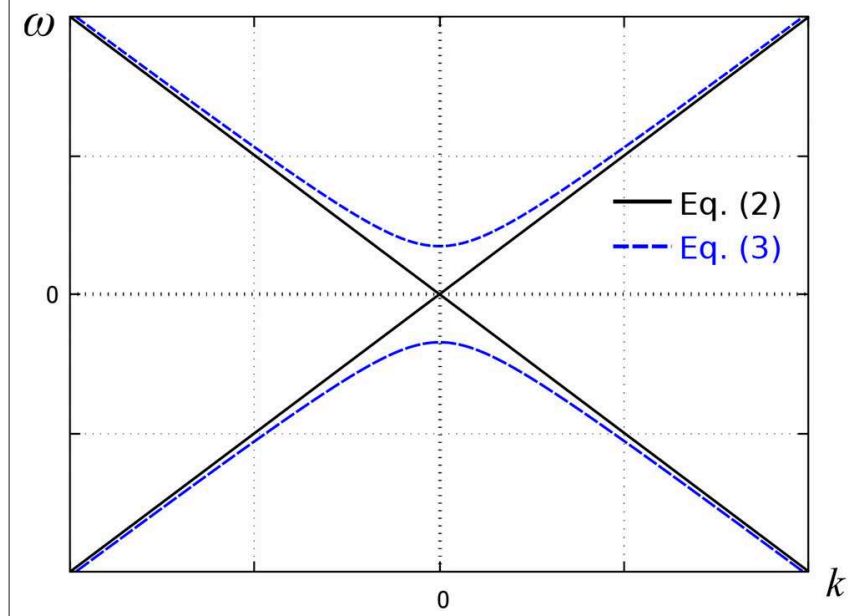

FIGURE 2 | Dispersion relation associated with Equations (2) and (3)

This is the essential property of the solitary wave. Here the single wave means the wave without undergoing any collisions with the other waves. Although such a property is common in linear cases, this should not be true in non-linear cases. If solitary waves preserve their shape and speed after a collision, the solitary waves holding a transparency is called the soliton. In particular, the terminology "soliton" is introduced by Zabusky and Kruskal [10]. Indeed, for the initially given sine waves, they are split into several solitary waves, and

- The Solitary wave moves stably by preserving momentum and shape even after the collisions.

- The Solitary wave possibly experiences a phase shift and time delay during the collision.

These are the properties to be satisfied by the soliton wave. That is, the solitary wave is called soliton if it satisfies the above properties. The transparency leading to the individuality is often called the particle-like property in the soliton theory. In particular, by comparing soliton waves before and after collision, there are no changes in the momentum and shape, but there is for the phase.

The equations holding the soliton as a solution are called the soliton equation, and the Korteweg-de-Vries equation ( $\mathrm{KdV}$ equation, for short) is known as a soliton equation. In a mathematical sense the concept of a solitary wave was initially studied by the KdV equation [11]

$$
\partial_{t} u+\alpha u \partial_{x} u+\partial_{x}^{3} u=0, \quad x \in \mathbb{R},
$$

where $\alpha$ is a real constant. In the second term $\alpha u$ plays a role of propagation speed (cf. Equation 2), so that the propagation speed depends on the state of the wave. This non-linear equation models the shallow water waves including both the non-linearity and the dispersive property, but not the dissipation leading to the non-unitary time evolution. It is worth noting here that the $\mathrm{KdV}$ equation is obtained by approximating the incompressible Navier-Stokes equation (for example, see Lamb [12]).
The plane wave (1) can be the solution at small amplitude oscillation limit, and then $\omega=c k-k^{3}$ is approximately satisfied. On the other hand, $\mathrm{KdV}$ equation admits some exact traveling wave solutions:

$$
u=\frac{3 c}{\alpha} \operatorname{sech}^{2}\left[\frac{\sqrt{c}}{2}(x-c t)\right]
$$

where $c$ means the speed of wave propagation. It is remarkable that Equation (6) holds the form of d'Alembert's solution for the wave equation. This solution corresponds to the solitary wave solution (one-soliton solution) whose amplitude depends on the propagation speed $c$. The solitary wave solution can hold the soliton property that has been examined by obtaining the exact two-soliton solution (Figure 1).

$$
u=\frac{72}{\alpha} \frac{3+4 \cosh (2 x-8 t)+\cosh (4 x-64 t)}{\{3 \cosh (x-28 t)+\cosh (3 x-36 t)\}^{2}}
$$

asymptotically equal to the superposition of two solitons for large $t$

$$
u=\frac{12 \kappa_{i}}{\alpha} \operatorname{sech}^{2}\left[\kappa_{i}\left(x-4 \kappa_{i}^{2} t\right)+\delta_{i}\right],
$$

where $i=1,2, \kappa_{1}=1, \kappa_{2}=2$, and $\delta_{i}$ are constants. The existence of the two-soliton solution ensures the existence of soliton in a given theoretical framework. In several equations the two-soliton solutions are extended to $\mathrm{N}$-soliton solutions (for example, see Scott et al. [13]).

\subsection{Non-linear Schrödinger Equation}

A typical soliton equation for non-relativistic quantum dynamics is the non-linear Schrödinger equation (NLS equation, for short). It reads

$$
i \partial_{t} u+\partial_{x}^{2} u+k|u|^{2} u=0,
$$

where a real number $k$ means the interaction constant, and also the height/depth of potential hill/well. Indeed, in the case of positive $k, V(u)=-k|u|^{2}$ provides a potential well. Indeed, it holds a solution

$$
\begin{aligned}
u(t, x) & =\sqrt{\frac{u_{e}^{2}-2 u_{e} u_{c}}{2 k}} \operatorname{sech}\left[\sqrt{\frac{u_{e}^{2}-2 u_{e} u_{c}}{4}}\left(x-u_{c} t\right)\right] \\
& \exp \left[i\left(u_{0} / 2\right)\left(x-u_{c} t\right)\right],
\end{aligned}
$$

where the amplitude of $u$ depends on the constant $k$, which is a specific feature arising from the angular speed $u_{c}$ and the wave propagation speed $u_{e}$. Contrary to the previous $\mathrm{KdV}$ equation, the amplitude is proportional to $k^{-1 / 2}$ and $u_{e}$. Consequently, the two factors have been considered to be essential to the soliton propagation: the dispersive property and the non-linearity.

\subsection{Sturm-Liouville Formalism}

Following Lax [14], the relation between the KdV and the Schrödinger type equations are understood by a simplified Sturm Liouville equation:

$$
L y:=\partial_{x}^{2} y-U(x, t) y=\lambda y,
$$


where the periodic boundary condition is imposed, for instance. This equation can be regarded as the Schrödinger equation with the potential $-\lambda+U(x, t)$. It is readily seen that

$$
\begin{aligned}
\partial_{t}(L y) & =\left(\partial_{t} L\right) y+L\left(\partial_{t} y\right)=\left(\partial_{t} \lambda\right) y+\lambda\left(\partial_{t} y\right)=-\left(\partial_{t} U(x, t)\right) y \\
& +\left(\partial_{x}^{2}-U(x, t)\right)\left(\partial_{t} y\right)
\end{aligned}
$$

leads to

$$
\left(\partial_{t} L\right) y=-\left(\partial_{t} U(x, t)\right) y .
$$

If $t$-independence of parameter $\lambda: \partial_{t} \lambda=0$ is further assumed,

$$
\begin{aligned}
& \left(\partial_{t} \lambda\right) y=-\left(\partial_{t} U(x, t)\right) y+L\left(\partial_{t} y\right)-\left(\partial_{t} \lambda y\right) \\
& \quad=-\left(\partial_{t} U(x, t)\right) y+L\left(\partial_{t} y\right)-\left(\partial_{t} L y\right) \\
& \quad=-\left(\partial_{t} U(x, t)\right) y+\left[L, \partial_{t}\right] y
\end{aligned}
$$

is obtained, where $[\cdot, \cdot]$ denotes the commutator product. After generalizing this equation as

$$
\left(\partial_{t} \lambda\right) y=-\left(\partial_{t} U(x, t)\right) y+[L, D] y
$$

the $\mathrm{KdV}$ equation with the potential $U$ and $\alpha=-6$ is obtained by $-\left(\partial_{t} U(x, t)\right)+[L, D]=0$ with $D=$ $f \partial_{x}^{3}+g \partial_{x}+h, g=-3 U f / 2$ and $h=-3\left(\partial_{x} u\right) f / 4$. Consequently, $\mathrm{KdV}$ and Schrödinger equations are associated not only with having a soliton solution, but with holding a common mathematical structure. An interesting reminder here is the relation between Schrödinger, Heisenberg, and interaction pictures in quantum field theory (for a textbook, see Fetter and Warecka [15]).

\section{SOLITONS IN NUCLEAR TDDFT}

\subsection{Many-Nucleon System}

Atomic nucleus is a finite-body many-nucleon system consisting of nucleons: protons and neutrons. Proton numbers range from 1 to 120 (at the present), and neutron numbers from 1, roughly, to 200. It is thought that almost 300 stable nuclei exist in nature, and the theoretical calculations such as nuclear density functional calculations simulate those nuclei as being sufficiently comparable to the experiments.

We are interested in the soliton propagation at the scale of atomic nuclei. The size of one nucleus ranges from $10^{-15}$ to $10^{-13}$ $\mathrm{m}$, and the corresponding energy is below several 10 s of $\mathrm{MeV}$ per nucleon. One of the unique features of the many-nucleon system is found in their finite-body property, which is quite different from most of many-electron systems being treated as infinite matter. This feature brings about the fact that the self-bound state (the localized wave) is naturally realized in both nature and theory of many-nucleon systems. Following the general usage of low-energy nuclear physics, the terminology "low-energy" is used for energy below $30 \mathrm{MeV}$ per nucleon. The relativistic effect plays a considerable role, only if the relative velocity of the collision is over $30 \%$ of the speed of light, and it roughly corresponds to the collision energy $30 \mathrm{MeV}$ per nucleon.
Ground states and some excited states of stable nuclei (in the following, self-bound nuclei) are classified to the localized selfbound system. Each self-bound system is the solitary wave in the soliton theory because it is satisfied that

- A self-bound nucleus moves stably without changing the shape

if there is no collision with the other nuclei/particles. Therefore, the existence of a solitary wave is trivially true for many-nucleon systems, where this issue should be examined by the non-linear framework with the ultimately determined density functional. In other words, all the self-bound nuclei are candidates of soliton. All we have to do to verify the soliton existence is to check that

- [Conditional] a nucleus moves stably by preserving momentum and shape even after the collisions.

- The nucleus possibly experiences the phase shift and the time delay.

The first condition is expected to be satisfied conditionally. On the other hand, the second condition is trivially satisfied in case of atomic nuclei, as phase shifts have been observed and theoretically calculated in nuclear reactions, as well as the time delay. One of the general motivations is to find a valid condition for the existence of the nuclear soliton.

\subsection{Theoretical Framework}

Among several theoretical models in nuclear physics, nuclear time-dependent density functional theory $[16,17]$ (TDDFT, for short), which describes the nuclear collision dynamics with the nucleon degree of freedom, is a unique theory including time dependence, non-linearity, and the dispersive property simultaneously. The solution of the TDDFT shows the unitary time evolution, which is preferable because of exact conservation of the total energy. The dispersive property is satisfied by the non-relativistic theory, while it is violated in the massless relativistic theories. In this context, we remind that the sineGordon equation is known as a soliton equation. Furthermore, it is worth noting here that, among sub-atomic theories except for the TDDFT, it is not easy to find a calculationally-feasible theoretical framework including the time-dependence. Note that the TDDFT is also called nuclear time-dependent Hartree-Fock theory, and nuclear reaction is often referred to as heavy-ion collision or ion collision. The theory with nucleon degree of freedom is called the microscopic treatment, because a nucleus is a smaller component that builds up a nucleus. The TDDFT is usually calculated in three-dimensional space, and the TDDFT have many stable localized stationary solutions corresponding to the self-bound nuclei. Non-linearity, dispersive property, and the unitary time-dependence realized in the TDDFT are preferable for examining the soliton existence. Furthermore, nuclear saturation property brings about rather universal shallow potential well with a depth of $50 \mathrm{MeV}$ at the deepest, whose environmental setting is ideal for the existence of certain kinds of shallow water waves.

Before moving on to the nuclear theoretical models, a few remarks are made on the multi-dimensional treatment. There is limited knowledge on the multi-dimensional soliton, where 
the shape of colliding waves plays more roles. In the multidimensional case, the soliton existence depends on whether the waves are spatially finite or not, and whether the waves are spherical or deformed. As a multi-dimensional version of $\mathrm{KdV}$ equation, Kadomtsev-Petviashvili equation (KP equation, for short) is known. In particular the multi-dimensional version of NLS equation (2) cannot have the self-bound solution, while the multi-dimensional NLS type equation

$$
\begin{aligned}
& i \partial_{t} u+\partial_{x}^{2} u+\partial_{y}^{2} u+k|u|^{2} u=u \partial_{x} v \\
& \partial_{x}^{2} v-\partial_{y}^{2} v=-2 \partial_{x}\left(|u|^{2}\right)
\end{aligned}
$$

is known to have the soliton (or dromion) solution instead [1820], where $v$ means the velocity potential. Roughly speaking, the addition of a non-linear term contributes to keeping the soliton property in this case.

\subsubsection{One-Dimensional Soliton Model}

Let us begin with reviewing the preceding work on soliton propagation in nuclear physics. In one-dimensional space, the Hamiltonian of $N$ bosons interacting through a $\delta$-force is represented by

$$
H=-\frac{1}{2} \sum_{i=1}^{N} \partial_{x_{i}}^{2}-v \sum_{i<j=1}^{N} \delta\left(x_{i}-x_{j}\right) .
$$

The corresponding stationary and non-stationary problems are known to be exactly solvable for bound states and for scattering states [21-26]. Application of the variational principle to

$$
\begin{gathered}
<\Psi\left|\partial_{t}-H\right| \Psi>=N \int d x\left(\psi^{*} i \partial_{t} \psi+\frac{1}{2} \psi^{*} \partial_{x}^{2} \psi\right. \\
\left.+\frac{v}{2}(N-1) \psi^{*} \psi^{*} \psi \psi\right)
\end{gathered}
$$

leads to

$$
i \partial_{t} \psi+\frac{1}{2} \partial_{x}^{2} \psi+\frac{v}{2}(N-1)|\psi|^{2} \psi=0,
$$

where $\Psi$ means the many-nucleon wave function and $\psi$ denotes single-nucleon wave function. The similarity to the NLS equation (2) is clear, so that the soliton solution follows. The static solution is

$$
\psi_{i}(x)=\frac{\sqrt{(N-1) v}}{2 \cosh ((N-1) v x / 2)}
$$

with the energy

$$
E_{H}=-\frac{N(N-1)^{2} v^{2}}{24}
$$

and the density

$$
\rho(x)=\frac{N(N-1) v}{4 \cosh ^{2}((N-1) v x / 2)} .
$$

For the $2 \mathrm{~N}$ particle case, a two-soliton solution is obtained. The two-soliton solution is represented by

$$
\begin{aligned}
& \psi(t, x)=\frac{\sqrt{2(N-1) v}}{2} e^{-(i / 2)\left(K^{2}-a^{2}\right) t} \\
& \quad \frac{e^{i K x}\left\{e^{-a(x-K t)}+\left(K^{2} /(K-i a)^{2}\right) e^{-a(3 x+K t)}\right\}+(K \leftrightarrow-K)}{1+2 e^{-2 a x} \cosh (2 a K t)-2 a^{2} e^{-2 a x} \operatorname{Re}\left(e^{2 i K x} /(K+i a)^{2}\right)+\left(K^{4} /\left(K^{2}+a^{2}\right)^{2}\right) e^{-4 a x}} .
\end{aligned}
$$

The existence of the two-soliton solution ensures the existence soliton in a given theoretical framework.

\subsubsection{Three-Dimensional Model}

A two-dimensional model is realized as the axial symmetric model in nuclear density functional theory dealing with finite quantum systems, and the axis of symmetry is taken as the collision axis in the time-dependent collision calculations. In this sense two dimensional calculation computes one-dimensional colliding motion along the center axis. One and two-dimensional models are toy models for simulating the collision, because the effect described by the outer product (vector product) cannot be rigorously incorporated. Consequently spin effect on the dynamics such as spin-orbit force effect cannot be rigorously treated in one and two dimensional models (cf. the representation of spin current $\boldsymbol{J}(\boldsymbol{r})$ in Equation (24)). Note that spin-orbit force in the non-relativistic framework arises from the special relativity theory. In particular the spin orbit force is wellknown to play a decisive role in the structure of nuclei (cf. magic numbers of nuclear structure [6]).

Let us consider the three-dimensional case. It is remarkable that the nuclear medium as a nucleon degree of freedom consists of two different kinds of fermions: protons and neutrons. In the following, the formalism of TDDFT [11, 16] for low-energy nuclear reactions are introduced based on [27], where the Skyrme interaction [28] is utilized as the effective nuclear force in most of the TDDFT calculations. The Skyrme interaction is a zerorange formalism of effective nucleon-nucleon interaction. The TDDFT with Skyrme type zero-range interaction is represented by several densities

$$
\begin{aligned}
\rho(\boldsymbol{r}) & =\sum_{i, \sigma}\left(\psi_{i}^{*}(\boldsymbol{r}, \sigma) \psi_{i}(\boldsymbol{r}, \sigma)\right), \\
\tau(\boldsymbol{r}) & =\sum_{i, \sigma}\left(\nabla \psi_{i}^{*}(\boldsymbol{r}, \sigma) \cdot \nabla \psi_{i}(\boldsymbol{r}, \sigma)\right), \\
\boldsymbol{j}(\boldsymbol{r}) & =\frac{1}{2 i} \sum_{i, \sigma}\left(\psi_{i}^{*} \nabla \psi_{i}(\boldsymbol{r}, \sigma)-\psi_{i} \nabla \psi_{i}^{*}(\boldsymbol{r}, \sigma)\right),
\end{aligned}
$$

and

$$
\begin{aligned}
& \boldsymbol{s}(\boldsymbol{r})=\sum_{i, \sigma, \sigma^{\prime}}\left(\psi_{i}^{*}(\boldsymbol{r}, \sigma) \psi_{i}\left(\boldsymbol{r}, \sigma^{\prime}\right)\left\langle\sigma|\hat{\sigma}| \sigma^{\prime}\right\rangle\right), \\
& \boldsymbol{T}(\boldsymbol{r})=\sum_{i, \sigma}\left(\nabla \psi_{i}^{*}(\boldsymbol{r}, \sigma) \cdot \nabla \psi_{i}\left(\boldsymbol{r}, \sigma^{\prime}\right)\left\langle\sigma|\hat{\sigma}| \sigma^{\prime}\right\rangle\right), \\
& \left.\boldsymbol{J}(\boldsymbol{r})=\frac{1}{2 i} \sum_{i, \sigma}\left(\psi_{i}^{*} \nabla \psi_{i}(\boldsymbol{r}, \sigma)-\psi_{i} \nabla \psi_{i}^{*}(\boldsymbol{r}, \sigma)\right) \times\left\langle\sigma|\hat{\sigma}| \sigma^{\prime}\right\rangle\right),
\end{aligned}
$$

where $\psi_{i}(\boldsymbol{r}, \sigma)$ and $\psi_{i}^{*}(\boldsymbol{r}, \sigma)$ are $i$-th single wave functions and its complex conjugate, respectively, $\rho(\boldsymbol{r}), \tau(\boldsymbol{r})$, and $\boldsymbol{j}(\boldsymbol{r})$ denote the density, the kinetic energy density, and the momentum density, respectively, and $\boldsymbol{s}(\boldsymbol{r}), \boldsymbol{T}(\boldsymbol{r})$, and $\boldsymbol{J}(\boldsymbol{r})$ stand for the spin density, the spin kinetic density, and the spin current density, respectively. Single wave functions depend on both spatial variable $r \in \mathbf{R}^{3}$ and the spin $\sigma$, while the spin dependence is summed up in each density. By assuming wave functions and densities as depending also on the time variable $t \in \mathbf{R}$, each single-nucleon satisfies the equation of the form.

$$
i \hbar \partial_{t} \psi_{i}(t, \boldsymbol{r}, \sigma)=h \psi_{i}(t, \boldsymbol{r}, \sigma)
$$


with

$$
\begin{aligned}
h \psi_{i}(t, \boldsymbol{r}, \sigma) & =\sum_{\sigma^{\prime}}\left[-\nabla \cdot \frac{\hbar^{2}}{2 m_{q}^{*}} \nabla \delta_{\sigma, \sigma^{\prime}}+U_{q}(\boldsymbol{r}) \delta_{\sigma, \sigma^{\prime}}\right. \\
& +\boldsymbol{V}_{q}(\boldsymbol{r}) \cdot\left\langle\sigma|\hat{\sigma}| \sigma^{\prime}\right\rangle+i C_{q}(\boldsymbol{r}) \cdot \nabla \delta_{\sigma, \sigma^{\prime}} \\
& \left.+i \boldsymbol{W}_{q}(\boldsymbol{r}) \cdot\left(\left\langle\sigma|\hat{\sigma}| \sigma^{\prime}\right\rangle \times \nabla\right)\right] \psi_{i}\left(t, \boldsymbol{r}, \sigma^{\prime}\right),
\end{aligned}
$$

where $h$ is the single-particle Hamiltonian, $m_{q}^{*}$ denotes the effective mass, and $U_{q}, V_{q}, C_{q}$, and $W_{q}$ mean the spin scalar potential, the spin vector potential, the current potential, and the spin orbit potential, respectively. The isospin index $q$ distinguishes protons $(q=p)$ from neutrons $(q=n)$. For realizing the fermionic statistical property, single wave functions are assumed to form the single Slater determinant, where this assumption is necessary to derive Equation (26). First, the nucleon-nucleon interaction is fully represented by the densities. Here the reason this formalism is called the nuclear TDDFT. Second, this formalism tells us that each single nucleon does not interact directly with the other nucleon, but with the force field described by the collectively summedup densities (23) and (24). This is the reason the nuclear TDDFT is claimed to be the theory, based on the meanfield description of the many-body interaction (in the same context, the nuclear TDDFT is also called the nuclear TDHF). Furthermore one-body dissipation with the unitarity appears mainly due to the internal excitation of nucleus. Note that the concept of one-body dissipation is a kind of dissipation, but it does not violate the unitarity of time evolution. The details are given by

$$
\begin{aligned}
& \frac{\hbar^{2}}{2 m_{q}^{*}}=\frac{\hbar^{2}}{2 m_{q}}+B_{3} \rho+B_{4} \rho_{q}, \\
& U_{q}(\boldsymbol{r})=2 B_{1} \rho+2 B_{2} \rho_{q}+B_{3}(\tau+i \nabla \cdot \boldsymbol{j})+B_{4}\left(\tau_{q}+i \nabla \cdot \boldsymbol{j}_{q}\right) \\
& +2 B_{5} \Delta \rho+2 B_{2} \Delta \rho_{q}+(2+\alpha) B_{7} \rho^{\alpha+1} \\
& +B_{8}\left\{\alpha \rho^{\alpha-1}\left(\rho_{n}^{2}+\rho_{p}^{2}\right)+2 \rho^{\alpha} \rho_{q}+B_{9}\left(\nabla \cdot \boldsymbol{J}+\nabla \cdot \boldsymbol{J}_{q}\right)\right\} \\
& +\alpha \rho^{\alpha-1}\left\{B_{12} s^{2}+B_{13} s_{n}^{2}\left(\boldsymbol{s}_{n}^{2}+\boldsymbol{s}_{p}^{2}\right)\right\} \\
& +\left[e^{2} \int \frac{\rho_{p}\left(\boldsymbol{r}^{\prime}\right)}{\left|\boldsymbol{r}-\boldsymbol{r}^{\prime}\right|} d \boldsymbol{r}^{\prime}-e^{2}\left(\frac{3 \rho_{p}}{\pi}\right)^{1 / 3}\right] \delta_{q, p}, \\
& \boldsymbol{V}_{q}(\boldsymbol{r})=B_{9}\left(\nabla \times \boldsymbol{j}+\nabla \times \boldsymbol{j}_{q}\right)+2 B_{10} \boldsymbol{s}+3 B_{11} \boldsymbol{s}_{q} \\
& +2 \rho^{\alpha}\left(2 B_{12} \boldsymbol{s}+2 B_{13} \boldsymbol{s}_{q}\right)+B_{9}\left(\nabla \times \boldsymbol{J}+\nabla \times \boldsymbol{J}_{q}\right), \\
& \boldsymbol{C}_{q}(\boldsymbol{r})=2 B_{3} \boldsymbol{j}+2 B_{4} \boldsymbol{j}_{q}-B_{9}\left(\nabla \times \boldsymbol{s}+\nabla \times \boldsymbol{s}_{q}\right), \\
& \boldsymbol{W}_{q}(\boldsymbol{r})=-B_{9}\left(\nabla \rho+\nabla \rho_{q}\right) \text {, }
\end{aligned}
$$

where a part shown inside the parenthesis $[\cdot]$ in $U_{q}(\boldsymbol{r})$ shows the Coulomb interaction acting only on protons. Thirteen different coefficients $\left(B_{1}, B_{2}, \cdots, B_{13}\right)$ must be determined, while they are reduced to only 10 parameters $\left(t_{0}, t_{1}, \cdots, x_{3}, \alpha\right)$. For the derivation of the above effective nuclear interaction, see [27, 29].

Although more than 100 parameter sets are proposed for the Skyrme-type effective nuclear interaction (the values for $\left.\left\{t_{0}, t_{1}, t_{2}, t_{3}, W_{0}, x_{0}, x_{1}, x_{2}, x_{3}, \alpha\right\}\right)$, the ultimate parameter set is

TABLE 1 | Parameter setting in the TDDFT.

(a) Reduced coefficients

$$
\begin{aligned}
& B_{1}=t_{0}\left(1+x_{0} / 2\right) / 2 \\
& B_{2}=-t_{0}\left(x_{0}+1 / 2\right) / 2 \\
& B_{3}=\left(t_{1}+t_{2}\right) / 4+\left(t_{1} x_{1}+t_{2} x_{2}\right) / 8 \\
& B_{4}=\left(t_{2}-t_{1}\right) / 8-\left(t_{2} x_{2}-t_{1} x_{1}\right) / 4 \\
& B_{5}=\left(t_{2}-3 t_{1}\right) / 16+\left(t_{2} x_{2}-3 t_{1} x_{1}\right) / 32 \\
& B_{6}=\left(3 t_{1}+t_{2}\right) / 32+\left(t_{1} x_{1}+t_{2} x_{2}\right) / 16 \\
& B_{7}=t_{3}\left(1+x_{3} / 2\right) / 12 \\
& B_{8}=-t_{3}\left(x_{3}+1 / 2\right) / 12 \\
& B_{9}=-W_{0} / 2 \\
& B_{10}=-t_{0} x_{0} / 4 \\
& B_{11}=-t_{0} / 4 \\
& B_{12}=-t_{3} x_{3} / 24 \\
& B_{13}=-t_{3} / 24
\end{aligned}
$$

The reduced coefficients (a) and a Skyrme parameter set (b) are shown. Among many parameter sets (models for the effective nuclear force), the SV-bas model is taken in this paper

not known to include such an existence. Here we take SV-bas parameter set (Table 1). The SV-bas parameter set is known well for reproducing the neutron skin thickness of heavy nuclei such as ${ }^{208} \mathrm{~Pb}$ (for a compilation of experimental and theoretical results, see von Neumann-Cosel [31]). The quality of SV-bas in some relevant heavy nuclei can be found in Iwata and Stevenson [7]. On the other hand, the description of light ions (helium isotopes) using SV-bas is also confirmed to be sufficiently good [32]. The pairing interaction is not introduced in the present density functional, as the collision energy of the present study is sufficiently high for pairing interaction not to play a significant role. Indeed, from an energetic point of view, the nuclear pairing is the effect of less than a few 100s of $\mathrm{keV}$ per nucleon. A set of equations (25), (26), and (27) are called the nuclear TDDFT or the nuclear TDHF equations. The nuclear TDDFT is known to reproduce the result rather sufficiently nowadays (for recent reviews, see [33-35]).

\subsection{Solitons in Many-Nucleon Systems 4.3.1. Similarity of Master Equations}

To verify the soliton existence, we begin by finding similarities between NLS (4) and the nuclear TDDFT. For one-dimensional cases, as the soliton solution has been obtained, Equation (18) is essentially identical to Equation (9). For three-dimensional cases, a term with $\hbar^{2} / 2 m_{q}^{*}$ in the TDDFT corresponds to the second term of the left hand side of Equation (9). Here we see that the TDDFT is a Schrödinger type equation. Meanwhile the nonlinear term $|u|^{2} u$ in Equation (9) corresponds to terms with the coefficients $B_{1}$ and $B_{2}$ (depending essentially on the parameter $t_{0}$ ). The terms with the coefficients $B_{7}$ and $B_{8}$ (depending essentially on the parameter $t_{3}$ ), which are known to be indispensable to reproduce the nuclear saturation properties [29], are also relevant, because they introduce additional fractional power contributions (cf. $\alpha$ in the Skyrme parameter set: the 
fractional power). The dominance of $t_{0}$ and $t_{3}$ terms has been confirmed for the binding energies of ${ }^{4} \mathrm{He}$ and ${ }^{8} \mathrm{He}$ [32], where the experimental binding energy is $28.30 \mathrm{MeV}$ and 31.40 for ${ }^{4} \mathrm{He}$ and ${ }^{8} \mathrm{He}$ respectively. No self-bound states of ${ }^{4} \mathrm{He}$ and ${ }^{8} \mathrm{He}$ are obtained if the $t_{0}$ term is turned off, and even in the presence of the $t_{0}$ term the calculated binding energies are at the order of $1,000 \mathrm{MeV}$ which are far from the realistic binding energy. More quantitatively, with respect to the bindings of ${ }^{4} \mathrm{He}$, a large binding (due to the attractive force property of $t_{0}$ term) at the order of $1,000 \mathrm{MeV}$ is obtained only by the $t_{0}$ term, it is substantially modified by the $t_{3}$ term (due to the repulsive force property of $t_{3}$ term) as $63.80 \mathrm{MeV}$, and the momentum density contribution ( $t_{1}$ term) reduces it to a realistic value of $27.71 \mathrm{MeV}$, where the binding energy calculated by including all the terms is $27.73 \mathrm{MeV}$. Note that the spin-orbit contribution is known to be important in the nuclear structure, but it does not play a prominent role in this case because ${ }^{4} \mathrm{He}$ is a spin-saturated system. A rough estimation tells us that the interaction part of the TDDFT with the SVbas model (the inhomogeneous term of non-linear Schrödinger type equation) is dominated by the $t_{0}$ and $t_{3}$ terms with the percentage:

$$
\frac{|63.80|}{|63.80|+|63.80-27.71|+|27.71-27.73|} \times 100=63.9 \%,
$$

where the amplitudes of the $t_{0}$ and $t_{3}$ terms, $t_{1}$ term and the other terms are estimated as $|63.80|,|63.80-27.71|$, and $\mid 27.71-$ 27.73 , respectively. Dominance of those terms in the nuclear density functional implies the validity of an energy-dependent soliton existence in which the $t_{0}$ and $t_{3}$ terms are responsible for the soliton existence and energy dependence, respectively. This similarity between NLS and the nuclear TDDFT provides a sound motivation to investigate the soliton propagation in nuclear TDDFT.

\subsubsection{Mechanism of Soliton Propagation in the TDDFT}

Some specific physics associated with many-nucleon systems are presented with respect to the soliton propagation. In threedimensional nuclear TDDFT, the existence of a solitary wave corresponds to the existence of self-bound stationary states. For low-energy nuclear reactions, fusion, deep inelastic collision, and collision-fission such as fusion-fission and quasi-fission may appear. Particularly, in case of fusion, the solitary waves are totally destroyed. It implies that a solitary wave cannot necessarily be the soliton, and the soliton existence is inevitably conditional. Let us begin with the collision between ${ }^{4} \mathrm{He}$ and ${ }^{8} \mathrm{He}$. Following the usage of nuclear reaction representation, the fusion reaction realized by collision between two self-bound nucleus ${ }^{4} \mathrm{He}$ (helium 4: 2 protons and 2 neutrons) and ${ }^{8} \mathrm{He}$ (helium 8: 2 protons and 6 neutrons) is represented by

$$
{ }^{8} \mathrm{He}+{ }^{4} \mathrm{He} \rightarrow{ }^{12} \mathrm{Be},
$$

where ${ }^{12} \mathrm{Be}$ (beryllium 12: 4 protons and 8 neutrons) is produced as a result of fusion reaction. Fusion reaction is generally an exothermic or endothermic reaction according to the total binding energy difference between reactants and products, where a chemical element iron $(Z=26)$ is the most stable element. On the other hand, if self-bound states ${ }^{4} \mathrm{He}$ and ${ }^{8} \mathrm{He}$ hold the soliton property,

$$
{ }^{8} \mathrm{He}+{ }^{4} \mathrm{He} \rightleftharpoons{ }^{4} \mathrm{He}+{ }^{8} \mathrm{He}
$$

takes place in which the total energy is conserved before and after the collision. In the context of reaction theory, the soliton property is included in a class of reactions with the time-reversal symmetry. The goal is to find the condition for the appearance of soliton events shown by Equation (29). The time reversal symmetry arises from the energy conservation, according to Noether's theorem, and the total energy is strictly conserved by the nuclear TDDFT framework. For each collision there are two controllable parameters: the relative velocity of collision (i.e., the collision energy) and the impact parameter of collision (usually denoted by $b \mathrm{fm}$ ). The condition for soliton existence is expected to be written by these two control parameters (i.e., the initial condition).

The soliton existence is confirmed by systematically calculating collision events. The fast charge equilibration mechanism, which is the generalized concept of fusion reaction, has been suggested to govern the mixing of protons and neutrons, including fusion and deep inelastic collisions [36]. Under the appearance of fast charge equilibration, the mixing between protons and neutrons is known to take place quite rapidly within the order of $10^{-22} \mathrm{~s}$ [36] that should be compared to the typical duration time of low-energy nuclear reactions $\left(\sim 10^{-20} \mathrm{~s}\right)$. The charge equilibrating wave propagates at around $90 \%$ of the fermi velocity of many-nucleon systems (corresponding to the speed of zero sound propagation [37]), so that the propagation speed of the charge equilibrating wave is roughly equal to a quarter of the speed of light. Soliton existence is false if we observe the charge equilibration. Consequently, the soliton propagation is realized by the competition between the fast charge equilibration and the transparency, originally due to a certain non-linearity $\left(t_{0}\right.$ and $t_{3}$ terms) of the TDDFT. The fast charge equilibrating wave has been confirmed to play a role only if the collision energy is below the charge equilibration upper-limit energy [36].

In the lower energies less than a few $\mathrm{MeV}$ per nucleon, nuclear fusion appears, and soliton cannot survive. In higher energies larger than $50 \mathrm{MeV}$ per nucleon, the nucleus breaks up into small pieces. On the other hand, the fast charge equilibration wave can exist only below the upper-limit energy, where the upper energy is almost $80 \%$ of the fermi energy which is in accordance with the fact that the propagation speed is almost $90 \%$ of the fermi velocity. In case of nuclear collisions, this energy is roughly equal to $10 \mathrm{MeV}$ per nucleon. This fact may contain a hint for finding the soliton existence condition, i.e., it is reasonable to search for the energy just above the fast charge equilibration upper-limit energy.

\subsubsection{Numerical Experiment}

The heuristic aspect of the numerical experiment plays important roles in the past and present soliton theory (e.g., Fermi-PastaUlam [38]). In this section systematic large-scale calculation 
TABLE 2 | Self-binding energies of the ground states [7] are calculated using the SV-bas effective nuclear force.

\begin{tabular}{lccc}
\hline$(\boldsymbol{A}, \boldsymbol{Z})$ & $\mathbf{B}\left({ }^{\boldsymbol{A}} \boldsymbol{Z}\right)$ & $\mathbf{B}\left({ }^{\boldsymbol{A}+\boldsymbol{4} Z)}\right.$ & $\mathbf{B}\left({ }^{\left.\mathbf{2}+{ }^{4} \mathbf{2 Z}\right)}\right.$ \\
\hline$(4,2)$ & $6.93(7.08)$ & $4.48(3.93)$ & $6.07(7.06)$ \\
$(16,8)$ & $8.21(7.98)$ & $7.84(7.57)$ & $8.75(8.58)$
\end{tabular}

Binding energy per nucleon (MeV) of the initial nuclei are compared to those of the intermediate fused system, and the corresponding experimental values are shown in parenthesis [40].

of the nuclear collision dynamics is carried out based on the TDDFT. Three-dimensional nuclear TDDFT calculations with the fully-introduced Skyrme-type interaction (10 parameters, as in the present calculations) were initiated in the 1990's [39]. On the other hand, many self-bound stationary states have already been calculated (theoretically found) by static calculations $\left(\partial \psi_{i}(t, r, \sigma)=0\right.$ in the TDDFT $)$ in the 1980's, and they are compared to the experiments. The impact parameter dependence of the soliton existence is systematically considered in three-dimensional calculations.

Before moving on to the main discussion, we briefly review the preceding results $[7,32]$. According to the calculations dealing with ${ }^{8} \mathrm{He}+{ }^{4} \mathrm{He},{ }^{20} \mathrm{O}+{ }^{16} \mathrm{O},{ }^{44} \mathrm{Ca}+{ }^{40} \mathrm{Ca}$, ${ }^{52} \mathrm{Ca}+{ }^{48} \mathrm{Ca},{ }^{104} \mathrm{Sn}+{ }^{100} \mathrm{Sn},{ }^{124} \mathrm{Sn}+{ }^{120} \mathrm{Sn}$ reactions, the energydependence of soliton emergence has been clarified only for lighter cases: ${ }^{8} \mathrm{He}+{ }^{4} \mathrm{He}$, and ${ }^{20} \mathrm{O}+{ }^{16} \mathrm{O}$ (cf. Figure 4 of Iwata and Stevenson [7]). For those lighter cases, a rough sketch of the energy-dependence is as follows: the soliton property is not so active for low energies less than a few $\mathrm{MeV}$ per nucleon; soliton property becomes active around $10 \mathrm{MeV}$ per nucleon, it achieves almost the perfect transparency around 10-30 MeV per nucleon, and the transparency again decreases for much higher energies (Figures 2, 3 of Iwata and Stevenson [7]). For a mass dependence, the most decisive factor for the soliton propagation in heavier collisions has been clarified to be the appearance of the fragmentation including the nucleon emissions (mostly neutron emission). On the other hand, massive momentum equilibration leading to the momentum equilibrium of each spatial point are activated around $80-100 \mathrm{MeV}$ per nucleon, and those energies are too high to be relevant to the suppression of nuclear soliton propagation. In this article, by focusing on the stability of $N=Z$ nucleus of the two colliding nuclei, we clarify the energy-dependent soliton property of ${ }^{4} \mathrm{He}$ and ${ }^{16} \mathrm{O}$.

The initial state of the non-stationary problem is prepared by the two stationary solutions. Let $A$ and $Z$ be mass number and the proton number of a colliding nucleus ${ }^{A} Z$. We consider a set of collisions:

$$
{ }^{A} Z+{ }^{A+4} Z
$$

as a generalization of Equation (28), where $(A, Z)=(4,2)$, $(16,8),(40,20),(48,20),(100,50)$, and $(120,50)$ are considered. Numerical solutions are obtained based on the finite difference method (for the details, see Maruhn et al. [41]). Threedimensional space is incremented by $1.0 \mathrm{fm}$, and the unit time step is set to one-third of $10^{-23} \mathrm{~s}$. Vacuum boxes are prepared as $24 \times 24 \times 24 \mathrm{fm}^{3}$ for the stationary problems, and as $64 \times$ $32 \times 32 \mathrm{fm}^{3}$ for the non-stationary problems. The center-ofmass of ${ }^{A} Z$ and ${ }^{A+4} Z$ are set to $(10, b / 2,0)$ and $(-10, b / 2,0)$, respectively, and the initial momentum of ${ }^{A} Z$ and ${ }^{A+4} Z$ to $\left(-\sqrt{2 M_{A} E_{K}}, 0,0\right)$ and $\left(\sqrt{2 M_{A+4} E_{K}}, 0,0\right)$, respectively. The parameter $b \mathrm{fm}$ imitates the impact parameter. The quantities $M_{A}$ and $M_{A+4}$ denote the mass of ${ }^{A} Z$ and ${ }^{A+4} Z$, respectively. The periodic boundary condition is imposed in the three-dimensional Cartesian grid.

In Table 2 the binding energies of initial states are shown to confirm the quality of the present calculations. The binding energy is not precisely the same as the experiment on the whole, but the difference is less than $15 \%$ for the lighter nuclei, and less than $5 \%$ is achieved for heavier nuclei. It simply shows the quality of the SV-bas parameter set. By changing $A, Z$ and the two control parameters, we can examine the mass and energy dependence of the final products. In particular, if the soliton wave is dominant, no nucleon transfer takes place between ${ }^{A} Z$ and ${ }^{A+4} Z$. If charge equilibrating wave is dominant, two neutron transfer from ${ }^{A+4} Z$ to ${ }^{A} Z$ is expected to be the most frequent reaction process. For an astrophysical comparison it is practical to define the typical temperature of collision using the kinetic energy per nucleon or the relative velocity of the collision. Based on the Bethe formula [42], the temperature of nuclear collision $[7,43]$ is defined by

$$
E_{K}=\left\{\begin{array}{lc}
\kappa T_{C} T & \left(T<T_{C}\right), \\
\kappa T^{2} & \left(T \geq T_{C}\right),
\end{array}\right.
$$

where $E_{K}$ is the total kinetic energy per nucleon, and $T_{C}=$ $\kappa^{-1}=7.2 \mathrm{MeV}$ is associated with the translation of the fermi energy of the many-nucleon system to the relativistic center-ofmass kinetic energy [44]. It shows that $E_{K}$ behaves linearly in low temperature and quadratically in high temperature. In this article the results are shown by the kinetic energy $E_{K}=1,2,3, \cdots$, $10 \mathrm{MeV}$.

According to the previous study [7], helium $(Z=2)$ and oxygen $(Z=8)$ isotopes have been proposed as candidates of nuclear soliton. This issue is examined from a stationary aspect. For nuclei with $Z \leq 20$, heavier nuclei become more stable than lighter nuclei, so that lighter nuclei tend to capture a neutron or proton easily. If this is also true for ${ }^{4} \mathrm{He}$ and ${ }^{16} \mathrm{O}$, they cannot hold the soliton property. For the verification of the proposed mechanism, the single neutron addition energy and single proton addition energy are approximately calculated using the energies of even-even nuclei. From an energetic point of view, the following quantities are calculated.

$$
\begin{aligned}
& \mathcal{E}_{n}(A, Z)=\frac{E(A+2, Z)-E(A, Z)}{2}, \\
& \mathcal{E}_{p}(A, Z)=\frac{E(A+2, Z+2)-E(A, Z)}{2},
\end{aligned}
$$

where $E(A, Z)$ means the binding energy for the ground state of a nucleus consisting of $Z$ protons and $A-Z$ neutrons. These quantities show the stability against adding one neutron $\left(\mathcal{E}_{n}(A, Z)\right)$ or one proton $\left(\mathcal{E}_{p}(A, Z)\right)$, respectively. Neutron capture or proton capture is not preferred if the value is positive. The upper panel of Figure 3 shows that the formation of the density-functional field (a kind of mean-field) is not enough 
for $Z \leq 8$ cases, and the directly-interacting few-body features are more important instead, where doubly-magic nuclei (helium and oxygen cases) show relatively good results comparable to experiments. The stability of ${ }^{4} \mathrm{He}$ and ${ }^{16} \mathrm{O}$ can be found in the lower panel of Figure 3. A nucleus is stable against the addition of nucleons, if both $\mathcal{E}_{n}(A, Z)$ and $\mathcal{E}_{p}(A, Z)$ are positive. ${ }^{4} \mathrm{He}$ and ${ }^{16} \mathrm{O}$ show the stability (lower panel of Figure 3 ), although heavier

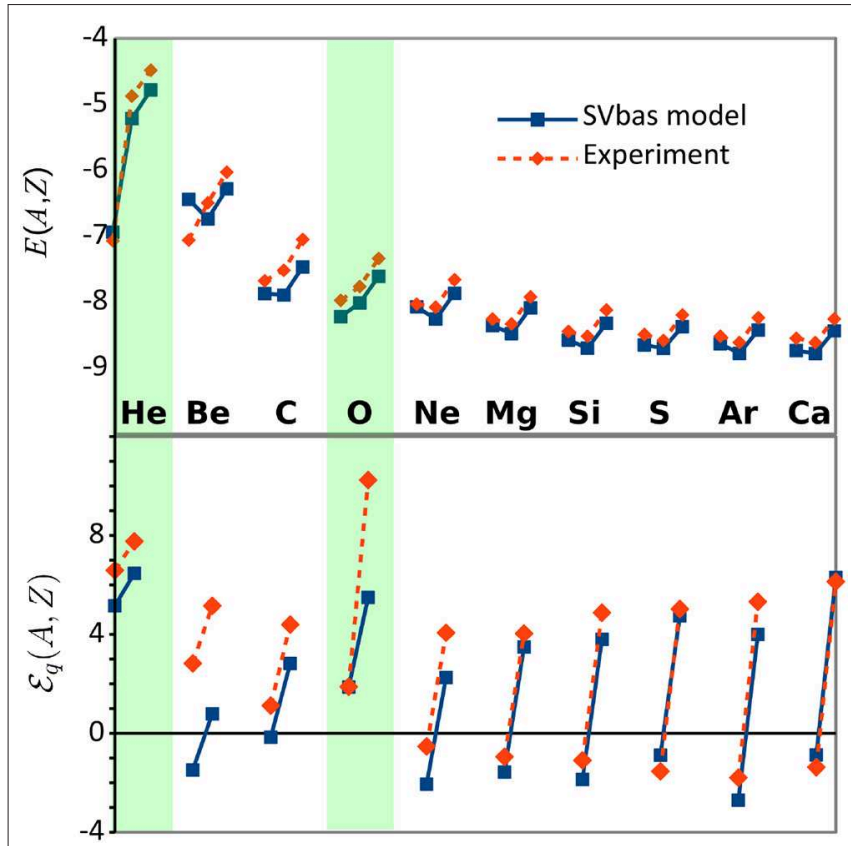

FIGURE 3 | Single particle energy and the corresponding energy differences are compared to experiments [40]. (Upper) For $Z \leq 20$ nuclei with $(A, Z)=(4,2)$, $(8,4), \cdots(40,20)$, the binding energies $E(A, Z), E(A+2, Z), E(A+2, Z+2)$ are shown by the connected lines in this order in each column. (Lower) The corresponding energy difference $\mathcal{E}_{n}(A, Z)$ and $\mathcal{E}_{p}(A, Z)$ for each nucleus ${ }^{Z} A$ is shown by the connected lines in this order. cases with $Z \geq 10$ will find a more stable bound system by adding neutrons. From an experimental point of view, ${ }^{4} \mathrm{He},{ }^{12} \mathrm{C}$, and ${ }^{16} \mathrm{O}$ are candidates of soliton, where ${ }^{8} \mathrm{Be}$ itself is known to be an unbound system even before comparing it to its neighbor nuclei. From a theoretical and experimental point of view, ${ }^{4} \mathrm{He}$ and ${ }^{16} \mathrm{O}$ are the candidates of soliton in which $\mathcal{E}_{q}(A, Z)$ values are positive. Consequently, the stability of soliton candidates ${ }^{4} \mathrm{He}$ and ${ }^{16} \mathrm{O}$ are confirmed with respect to the stability of the stationary state in comparison to the neighbors.

A time evolution of ${ }^{8} \mathrm{He}+{ }^{4} \mathrm{He}$ collision is shown in Figure 4. ${ }^{8} \mathrm{He}$ is coming from the left hand side, and ${ }^{4} \mathrm{He}$ is moving from the right hand side. It forms a rotating merged system around $t=28 / 3 \times 10^{-22} \mathrm{~s}$, and it is separated into two fragments as a result of collision. As for Figure 4, the collision energy is selected as the lowest energy at which the soliton wave component starts to appear. That is, taking this energy as the standard energy, for lower energies soliton cannot exist, and fusion reaction takes place; for higher energies almost perfect transparency with respect to both mass and momentum is realized. It is a non-central collision $(b \neq 0)$ in which the axial symmetry along the collision axis is essentially violated. The shape (more precisely, non-spherical property of the density and momentum distribution) is an important factor in the multidimensional case, where a less-symmetric shape of the merged system is introduced by the parameter $b$. In addition to the internal excitation, a part of the total energy is delivered to the angular momentum of each nucleus in case of multi-dimensional and $b \neq 0$ cases. To a certain degree, the appearance of rotational motion of the merged nucleus is a specific factor for the multidimensional soliton existence.

The detail of the nucleon transfer depends on the impact parameter, therefore on the shape and geometry. For ${ }^{8} \mathrm{He}+{ }^{4} \mathrm{He}$ collision transferred nucleon numbers are shown in Figure 5 , where the impact parameter dependence is shown in an energydependent manner. By increasing the energy, nucleon transfer starts to disappear after $E_{K}=7.50 \mathrm{MeV}$. Indeed, for cases with

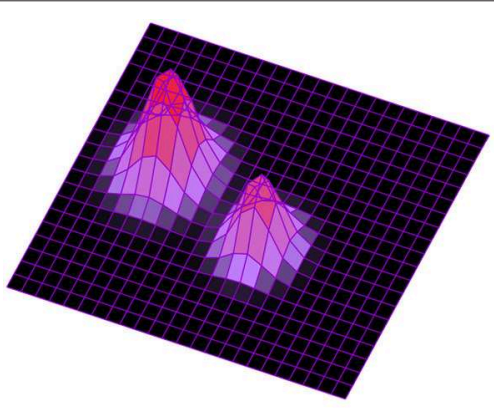

$t=8 / 3 \times 10^{-22} \mathrm{~s}$

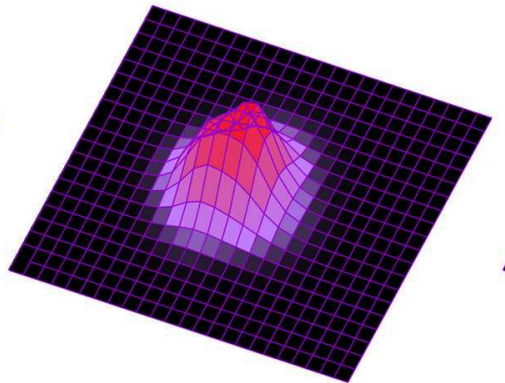

$t=28 / 3 \times 10^{-22} \mathrm{~s}$

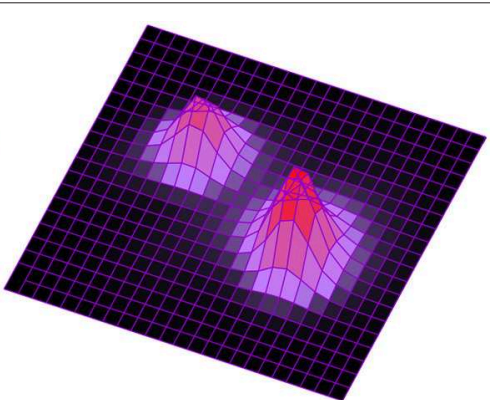

$t=48 / 3 \times 10^{-22} \mathrm{~s}$

FIGURE 4 | Imperfect soliton including the spin degree of freedom, fermionic statistical property, the multi-dimensionality, and the effect due to the non-central collision. Time evolution of ${ }^{8} \mathrm{He}+{ }^{4} \mathrm{He}$ for $E_{K}=7.50 \mathrm{MeV}$ and $b=3.0 \mathrm{fm}$ are shown. The collision energy is around the upper-limit energy of fast charge equilibration [36]. That is, by increasing the energy, the transparent component becomes dominant. For better sights, time evolution of total density is depicted by projecting them on the reaction plane $(z=0)$. The density is plotted on the vertical axis taken from 0 to $0.6 \mathrm{fm}^{-1}$, where the horizontal area is fixed to $(x, y)=24 \times 20 \mathrm{fm}{ }^{2}$. In this situation, 0.29 protons are transferred from ${ }^{4} \mathrm{He}$ to ${ }^{8} \mathrm{He}$, and 0.26 neutrons are transferred from ${ }^{8} \mathrm{He}$ to ${ }^{4} \mathrm{He}$, where we can find a weak effect of dual-way type charge equilibration [45] leading to the contamination of pure soliton. The self-bound property of ${ }^{8} \mathrm{He}$ and ${ }^{4} \mathrm{He}$ contributes to recovering the original shape if the transparencies of both mass and momentum is sufficiently high (kinetic energy loss is less than $5 \mathrm{MeV}$, see Figure $\mathbf{3}$ ). 

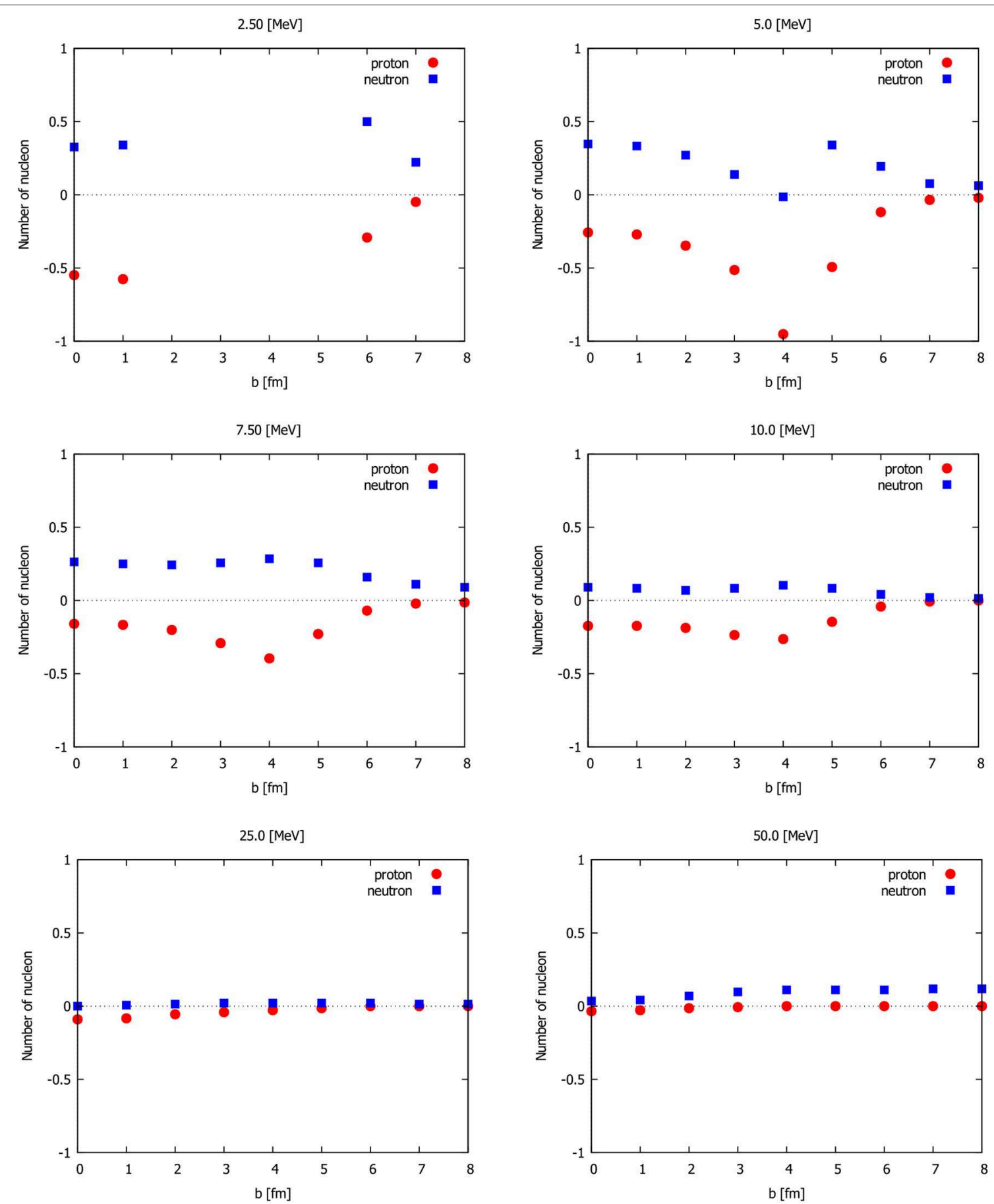

FIGURE 5 | For collisions ${ }^{8} \mathrm{He}+{ }^{4} \mathrm{He}$, transferred nucleon from ${ }^{8} \mathrm{He}$ to ${ }^{4} \mathrm{He}$ are shown depending on the relative velocity of the collisions. The impact parameter dependence with six different energies $E_{K}$ are shown. Red circles show the amounts of neutron transfer, and the blue squares show those of proton transfer. In a low energy case with $E_{K}=2.50 \mathrm{MeV}$ and $b=2,3,4,5 \mathrm{fm}$, fusion appears.

$E_{K}=7.50,10.0,25.0,50.0 \mathrm{MeV}$, the expectation value for the number of nucleon transfers are always less than 0.50 , so that the soliton wave is concluded to be dominant in those cases.

With respect to the quantum mechanical observation, the calculated results are statistically summed up for a given collision energy (a given relative velocity). Indeed, we cannot divide possible events by the impact parameter. Using the concept of geometric cross section [46], the numbers of total cross sections of all the inelastic events (events with touching between two nuclei) for a given collision energy $E_{K}$ is 
TABLE 3 | The observation probability [\%] of soliton state of ${ }^{4} \mathrm{He}$ calculated by $\mathcal{P}_{m}\left(E_{K}\right)$ and $\mathcal{P}_{p}\left(E_{K}\right)$ for given collision energies $E_{K}$

\begin{tabular}{lcccccc}
\hline & \multirow{3}{*}{$\mathcal{P}_{\boldsymbol{m}}\left(E_{K}\right)$} & & & \multicolumn{3}{c}{$\mathcal{P}_{p}\left(E_{K}\right)$} \\
\cline { 1 - 2 } \cline { 5 - 7 }$E_{K}[\mathrm{MeV}]$ & Proton & Neutron & & $E_{K}[\mathrm{MeV}]$ & Proton & Neutron \\
\hline 2.50 & 68.3 & 61.4 & & 2.50 & 74.5 & 72.5 \\
5.00 & 75.2 & 69.4 & & 5.00 & 60.2 & 56.2 \\
7.50 & 86.5 & 82.1 & & 7.50 & 64.6 & 62.4 \\
10.0 & 90.7 & 95.0 & & 10.0 & 76.9 & 73.7 \\
25.0 & 98.5 & 98.2 & & 25.0 & 95.0 & 93.5
\end{tabular}

Since the positions of center-of-mass are not so different for protons and neutrons in the present cases, the momentum transfer is shown as the total momentum transfer of all nucleons. According to the preceding study, the soliton is suggested to exist around $E_{K}=25.0 \mathrm{MeV}[7]$.

calculated by

$$
\begin{aligned}
\pi(1.50)^{2} T\left(b_{0}, E_{K}\right) & +\sum_{\substack{\left.\left.b_{i}=1 \\
-\pi\left(b_{i}-0.50\right)^{2}\right) T\left(b_{i}, E_{K}\right)\right)}}^{10}\left(\pi\left(b_{i}+0.50\right)^{2}\right.
\end{aligned}
$$

where $b_{i}$ fm imitates the impact parameter; $T\left(b_{i}, E_{K}\right)=1$ for touched cases, and $T\left(b_{i}, E_{K}\right)=0$ for untouched cases. As readily understood by the definition, events with a large impact parameter hold a larger cross section. The rate of transparent events measured by the particle transparency for a given collision energy is calculated by

$$
\begin{aligned}
& \mathcal{P}_{m}\left(E_{K}\right)=1- \\
& \frac{\left.\left.\pi(1.50)^{2} \mid N\left(b_{0}, E_{K}\right)\right)\left|+\sum_{b_{i}=1}^{10}\left(\pi\left(b_{i}+0.50\right)^{2}-\pi\left(b_{i}-0.50\right)^{2}\right)\right| N\left(b_{i}, E_{K}\right)\right) \mid}{\left.\pi(1.50)^{2} T\left(b_{0}, E_{K}\right)+\sum_{b_{i}=1}^{10}\left(\pi\left(b_{i}+0.50\right)^{2}-\pi\left(b_{i}-0.50\right)^{2}\right) T\left(b_{i}, E_{K}\right)\right)}
\end{aligned}
$$

where $N\left(b_{i}\right)$ is the transferred nucleon numbers. This definition can be regarded as the probability, in which $\left|N\left(b_{i}, E_{K}\right)\right|$ is taken as 1 for $\left|N\left(b_{i}, E_{K}\right)\right|>1$. According to this treatment, $\mathcal{P}_{m}\left(E_{K}\right)$ can be regarded as the probability for the particle transparency. Using the same definition in the geometric cross section, the transferred momentum rate is calculated. The rate of transparent events measured by the momentum transparency for a given collision energy is calculated by

$$
\begin{aligned}
& \mathcal{P}_{p}\left(E_{K}\right)=1- \\
& \frac{\left.\left.\pi(1.50)^{2} \mid M\left(b_{0}, E_{K}\right)\right)\left|+\sum_{b_{i}=1}^{10}\left(\pi\left(b_{i}+0.50\right)^{2}-\pi\left(b_{i}-0.50\right)^{2}\right)\right| M\left(b_{i}, E_{K}\right)\right) \mid}{10} \\
& \left.\pi(1.50)^{2} T\left(b_{0}, E_{K}\right)+\sum_{b_{i}=1}^{10}\left(\pi\left(b_{i}+0.50\right)^{2}-\pi\left(b_{i}-0.50\right)^{2}\right) T\left(b_{i}, E_{K}\right)\right)
\end{aligned}
$$

where $-1 \leq M\left(b_{i}\right) \leq 1$ is the transferred momentum divided by the initial momentum.
The soliton probability for all the possible collisions at given energies is summarized in Table 3. To find the soliton events at the energy just above the charge equilibration upper limit energy, it is reasonable to focus on $E_{K}=7.5 \mathrm{MeV}$ and $E_{K}=$ $10.0 \mathrm{MeV}$ cases. Indeed, for the reference case $E_{K}=25 \mathrm{MeV}$, single nucleon emission (neutron emission in most cases) took place during and after the collision, and the shapes are not well-conserved.

In case of helium collisions, almost $90 \%$ of the reaction is mass transparent for $E_{K}=10.0 \mathrm{MeV}$, and almost $80 \%$ is for $E_{K}=7.5 \mathrm{MeV}$. The corresponding momentum transparency rate is $89.6 \%$ for $E_{K}=10.0 \mathrm{MeV}$, and $92.1 \%$ for $E_{K}=7.5$ $\mathrm{MeV}$. Consequently, the probability for finding the soliton events calculated by the product

$$
\mathcal{P}_{p}\left(E_{K}\right) \mathcal{P}_{p}\left(E_{K}\right)
$$

are $81 \%$ for $E_{K}=10.0 \mathrm{MeV}$, and $76 \%$ for $E_{K}=7.5 \mathrm{MeV}$. In case of oxygen collisions, almost $70 \%$ of the reaction is mass transparent for $E_{K}=10.0 \mathrm{MeV}$, and almost $60 \%$ is for $E_{K}=7.5 \mathrm{MeV}$. The corresponding momentum transparency rate is $92.4 \%$ for $E_{K}=10.0 \mathrm{MeV}$, and $87.8 \%$ for $E_{K}=7.5$ $\mathrm{MeV}$. Consequently, the probability for finding the soliton events are $65 \%$ for $E_{K}=10.0 \mathrm{MeV}$, and $53 \%$ for $E_{K}=7.5 \mathrm{MeV}$. In both cases with helium and oxygen collisions, the cross section for soliton events is at the order of $1,000 \mathrm{mb}$ (millibarn). The soliton observation probabilities are larger than 50 $\%$, so that those collisions tend to be observed as the soliton time-reversible events.

\section{SUMMARY}

The soliton existence is nothing but the existence of perfect transparency, therefore the existence of perfect fluidity. The theoretical evidence for the imperfect nuclear soliton existence has been presented for the first time in a realistic setting. As a result, ${ }^{4} \mathrm{He}$ is concluded to be a candidate of nuclear soliton. ${ }^{16} \mathrm{O}$ also behaves like a soliton to a lesser degree. As the fermi energy can be different for different fermions, the present study brings about new insights on the validity of the different physics in different scales. Through the competition relation, the existence of nuclear soliton has been shown to depend essentially on the fermi energy of many-nucleon systems. An essential role of non-linearity in the formation of our material world is understood by the soliton propagation, since nucleon degree of freedom is related to the synthesis of chemical elements $(\mathrm{H}, \mathrm{He}$, $\mathrm{Li}, \mathrm{Be}, \cdots)$. In conclusion, ${ }^{4} \mathrm{He}$ and ${ }^{16} \mathrm{O}$ are suggested to be candidates for nuclear soliton. From an applicational point of view, the soliton property of these nuclei will be utilized for the preservation of ${ }^{4} \mathrm{He}$ matter, the condensation of ${ }^{4} \mathrm{He}$ matter, and the production/synthesis of certain nucleus (by adding several ${ }^{4} \mathrm{He}$ intentionally).

As seen in the competition mechanism between soliton wave propagation and charge equilibrating wave propagation, the conditions for the soliton propagation depend essentially on the fermi energy of the fermionic quantum system. Accordingly, 
there are some conjectures that need to be confirmed in the near future. In quantum systems,

1. The fermionic soliton exists in different scales in different ways, as the fermi energy is determined by fixing effective degrees of freedom.

2. The solitons between fermionic and bosonic systems are essentially different.

3. The general and special relativity effects change the soliton existence.

4. Another type of the soliton appears in the event when fermions and bosons are tightly correlated (e.g., supersymmetric systems).

5. Another type of the soliton exists in anionic systems.

Where the first, second, and third conjectures are partly studied in this article. The third and fifth conjectures are also associated with clarifying the difference compared to the Maxwellian systems or anionic systems. That is, as in the present research, the soliton propagation in quantum systems should be examined by considering the spin degree of freedom and multidimensional spatial-degree of freedom. The fourth conjecture is expected to play a role in clarifying and identifying the theory of everything.

As a closing remark some related open problems should be pointed out. Although there are several unknown and interesting topics in nuclear physics, we focus on the soliton propagation in many-nucleon systems.

- Show the similarity/difference between the soliton "perfect fluidity" and the bosonic "superfluidity."

- Show quantitatively that the soliton propagation is suppressed/enhanced by the interaction terms other than $t_{0^{-}}$ and $t_{3}$-terms.

- Show the soliton existence probability under the influence of many-body dissipation (cf. one-body dissipation in the main text).

\section{REFERENCES}

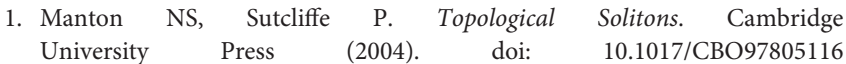
17034

2. Weinberg EJ. Classical Solutions in Quantum Field Theory. Cambridge University Press (2012).

3. Lee TD, Pang Y. Nontopological solitons. Phys Rep. (1992) 221:251. doi: 10.1016/0370-1573(92)90064-7

4. Ablowitz MJ. Nonlinear Dispersive Waves: Asymptotic Analysis and Solitons. Cambridge University Press (2011).

5. Greiner W, Maruhn JA. Nuclear Models. Berlin, Heidelberg: Springer-Verlag (1996). doi: 10.1007/978-3-642-60970-1

6. Ring P, Schuck P. The Nuclear Many-Body Problem. Berlin, Heidelberg: Springer-Verlag (1980). doi: 10.1007/978-3-642-61852-9

7. Iwata Y, Stevenson P. Conditional recovery of time-reversal symmetry in many nucleus systems. New J Phys. (2019) 21:043010. doi: 10.1088/1367-2630/ab0e58

8. Bjorken JD, Drell SD. Relativistic Quantum Mechanics. New York, NY: McGraw-Hill Companies (1998).

9. Scott Russell J. On Certain Effects Produced on Sound by the Rapid Motion of the Observer. Report of the Eighteenth Meeting of the British (1848).
- Find the charge-parity symmetry breaking reaction in terms of the conditional/unconditional soliton existence (conditional/unconditional time-reversal symmetry).

These things will clarify the role of the imperfect soliton in manynucleon systems. This kind of soliton should be different from the solitons in many-quark systems.

\section{DATA AVAILABILITY STATEMENT}

All datasets generated for this study are included in the article/supplementary material.

\section{AUTHOR CONTRIBUTIONS}

The author confirms being the sole contributor of this work and has approved it for publication.

\section{FUNDING}

This work was partially supported by JSPS KAKENHI Grant No. 17K05440. Numerical computation was carried out at Yukawa Institute Computer Facility of Kyoto University, and a workstation system at Kansai University.

\section{ACKNOWLEDGMENTS}

This article is a transcript of the seminar "The fundamentals of soliton theory and its applcation to many-nucleon systems" at the department of physics, Kyoto University, Japan on Nov. 21, 2019. Fruitful comments from Profs. K. Hagino, Y. Kanada-Enyo, N. Itagaki, K. Yoshida of Kyoto University and the other participants are acknowledged. The author is grateful to Editors Profs. C. Simenel, P. Stevenson, D. Lacroix, L. Guo, and N. Schank for giving him a chance to write this article.

10. Zabusky NJ, Kruskal MD, Interaction of "Solitons" in a collisionless plasma and the recurrence of initial states. Phys Rev Lett. (1965) 15:240. doi: 10.1103/PhysRevLett.15.240

11. Korteweg DJ, de Vries G, On the change of form of long waves advancing in a rectangular canal, and on a new type oflong stationary waves. Philos Mag. (1895) 39:422-43. doi: 10.1080/14786449508620739

12. Lamb GL Jr. Elements of Soliton Theory. New York, NY: John Wiley \& Sons (1980). Available online at: https://www.cambridge.org/ core/journals/laser-and-particle-beams/article/g-l-lambjr-elementsof-soliton-theory-john-wiley-sons-new-york-1980-302-pages/ B7746782CA289EFE25F3A17BD6DB3DB2

13. Scott W, Chu FY, Machlaughlin DW. The soliton: a new concept in applied science. Proc IEEE. (1973) 61:1443. doi: 10.1109/PROC.1973.9296

14. Lax PD. Integrals of nonlinear equations of evolution and solitary waves. Pure Appl Math. (1968) 21:467. doi: 10.1002/cpa.3160210503

15. Fetter L, Warecka JD. Quantum Theory of Many-Particle Systems. Mineola, NY: Dover (2003).

16. Dirac PAM. Note on exchange phenomena in the thomas atom. Proc Camb Philos Soc. (1930) 26:376. doi: 10.1017/S0305004100016108

17. Engel YM, Brink DM, Goeke K, Krieger SJ, Vautherin D. Hartree-Fock calculations with Skyrme's interaction. I. Spherical nuclei. Nucl Phys A. (1975) 249:215. doi: 10.1016/0375-9474(75)90184-0 
18. Davey Stewartson K. On three-dimensional packets of surface waves. Proc $R$ Soc Lond A. (1974) 338:101. doi: 10.1098/rspa.1974.0076

19. Boiti M, Leon JJP, Penpinelli F. A new spectral transform for the Davey-Stewartson I equation. Phys Lett A. (1989) 141:101. doi: 10.1016/0375-9601(89)90767-6

20. Hietarinta J, Hirota R. Multidromion solutions to the Davey-Stewartson equation. Phys Lett A. (1990) 145:237. doi: 10.1016/0375-9601(90)90357-T

21. Bethe HA. On the theory of metals. $Z$ Phys. (1931) 71:205. doi: $10.1007 / \mathrm{BF} 01341708$

22. Dolan L. Scattering of quantized solitary waves in the cubic Schrödinger equation. Phys Rev D. (1976) 13:528. doi: 10.1103/PhysRevD.13.528

23. McGuire JB. Study of exactly soluble one dimensional N body problems. $J$ Math Phys. (1964) 5:622. doi: 10.1063/1.1704156

24. Yang CN. Some exact results for the many-body problem in one dimension with repulsive delta-function interaction. Phys Rev Lett. (1967) 19:1312. doi: 10.1103/PhysRevLett.19.1312

25. Yoon B, Negele JW. Time-dependent Hartree approximation for a onedimensional system of bosons with attractive dfunction interactions. Phys Rev A. (1977) 16:1451. doi: 10.1103/PhysRevA.16.1451

26. Zakharov VE, Shabat AB. Exact theory of two-dimensional self-focusing and onedimensional self-modulation of waves in nonlinear media. Sov Phys JETP. (1972) 34:62

27. Bonche P, Flocard H, Heenen PH. Solution of the Skyrme HF + BCS equation on a 3D mesh. Comput Phys Commun. (2005) 171:49. doi: 10.1016/j.cpc.2005.05.001

28. Skyrme THR. The effective nuclear potential. Philos Mag. (1956) 1:1043; Nucl. Phys. (1956) 9:615. doi: 10.1080/14786435608238186

29. Vautherin D, Brink DM. Hartree-fock calculations with Skyrme's interaction. I. Spherical Nuclei. Phys Rev. (1972) C5:626. doi: 10.1103/PhysRevC.5.626

30. Klüpfel P, Reinhard P-G, Bürvenich TJ, Maruhn JA. Variations on a theme by Skyrme: a systematic study ofadjustments of model parameters. Phys Rev C. (2009) 79:034310. doi: 10.1103/PhysRevC.79. 034310

31. von Neumann-Cosel P. Complete electric dipole response in $120 \mathrm{Sn} 208 \mathrm{~Pb}$ implications for neutron skin symmetry energy. J Phys Conf Ser. (2015) 590:012017. doi: 10.1088/1742-6596/590/1/012017

32. Iwata Y. Energy-dependent existence of soliton in the synthesis of chemical elements. Mod Phys Lett A. (2015) 30:155008. doi: 10.1142/S0217732315500881

33. Simenel C, Umar AS. Heavy-ions collisions and fission dynamics with the time-dependent Hartree-Fock theory and its extension. Prog Part Nucl Phys. (2018) 103:19. doi: 10.1016/j.ppnp.2018.07.002
34. Stevenson P, Barton MC. Low-energy heavy-ion reactions and effective forces Prog Part Nucl Phys. (2019) 104:142. doi: 10.1016/j.ppnp.2018.09.002

35. Sekizawa K. TDHF theory and its extensions for the multinucleon transfer reaction: a mini review. Front Phys. (2019) 7:20. doi: 10.3389/fphy.2019.00020

36. Iwata Y, Otsuka T, Maruhn JA, Itagaki N. Suppression of charge equilbration leading to the synthesis of exotic nuclei. Phys Rev Lett. (2010) 104:252501. doi: 10.1103/PhysRevLett.104.252501

37. Iwata Y. Zero sound propagation in femto-scale quantum liquids. J Mod Phys. (2012) 6:476. doi: 10.4236/jmp.2012.36064

38. Fermi E, Pasta JR, Ulam SM. Studies of Nonlinear Problems. I. Los Alamos report LA-1940 (1955). doi: 10.2172/4376203

39. Kim K-H, Otsuka T, Bonche P. Three-dimensional TDHF calculations for reactions of unstable nuclei. $J$ Phys G. (1997) 23:1267. doi: 10.1088/0954-3899/23/10/014

40. Nudat 2.7. Available online at: www.nndc.bnl.gov/nudat2/

41. Maruhn JA, Reinhard P-G, Stevenson PD, Umar AS. The TDHF code Sky3D. Comput Phys Commun. (2014) 185:2195: doi: 10.1016/j.cpc.2014.04.008

42. Bethe HA. Nuclear physics nuclear dynamics B theoretical. Rev Mod Phys. (1937) 9:69. doi: 10.1103/RevModPhys.9.69

43. Fröbrich P, Lipperheide R. Theory of Nuclear Reactions. Oxford: Oxford University Press (1996)

44. Iwata Y. Time-scaled scenario of low-energy heavy-ion collisions. J Phys Conf Ser. (2013) 445:012017. doi: 10.1088/1742-6596/445/1/012017

45. Iwata Y, Otsuka T, Maruhn JA, Itagaki N. Geometric classification of nucleon transfer at moderate low-energies. Nucl Phys A. (2010) 836:108. doi: 10.1016/j.nuclphysa.2010.01.242

46. Iwata Y. Reaction cross sections for time-dependent density functional calculations. In: Fission and Properties of Neutron-Rich Nuclei - Proceedings of the Fifth International Conference on ICFN5. Singapore: World Scientific. 643-649. Available online at: https://www.worldscientific.com/ worldscibooks/10.1142/8910

Conflict of Interest: The author declares that the research was conducted in the absence of any commercial or financial relationships that could be construed as a potential conflict of interest.

Copyright (C) 2020 Iwata. This is an open-access article distributed under the terms of the Creative Commons Attribution License (CC BY). The use, distribution or reproduction in other forums is permitted, provided the original author(s) and the copyright owner(s) are credited and that the original publication in this journal is cited, in accordance with accepted academic practice. No use, distribution or reproduction is permitted which does not comply with these terms. 